\title{
Insights into the CRISPR/Cas system of Gardnerella vaginalis
}

\author{
Milda Pleckaityte*, Milda Zilnyte and Aurelija Zvirbliene
}

\begin{abstract}
Background: Gardnerella vaginalis is identified as the predominant colonist of the vaginal tracts of women diagnosed with bacterial vaginosis (BV). G. vaginalis can be isolated from healthy women, and an asymptomatic BV state is also recognised. The association of $G$. vaginalis with different clinical phenotypes could be explained by different cytotoxicity of the strains, presumably based on disparate gene content. The contribution of horizontal gene transfer to shaping the genomes of $G$. vaginalis is acknowledged. The CRISPR loci of the recently discovered CRISPR/Cas microbial defence system provide a historical view of the exposure of prokaryotes to a variety of foreign genetic elements.

Results: The CRISPR/Cas loci were analysed using available sequence data from three G. vaginalis complete genomes and $18 \mathrm{G}$. vaginalis draft genomes in the NCBI database, as well as PCR amplicons of the genomic DNA of 17 clinical isolates. The cas genes in the CRISPR/Cas loci of G. vaginalis belong to the E. coli subtype. Approximately $20 \%$ of the spacers had matches in the GenBank database. Sequence analysis of the CRISPR arrays revealed that nearly half of the spacers matched $G$. vaginalis chromosomal sequences. The spacers that matched G. vaginalis chromosomal sequences were determined to not be self-targeting and were presumably neither constituents of mobile-element-associated genes nor derived from plasmids/viruses. The protospacers targeted by these spacers displayed conserved protospacer-adjacent motifs.

Conclusions: The CRISPR/Cas system has been identified in about one half of the analysed G. vaginalis strains. Our analysis of CRISPR sequences did not reveal a potential link between their presence and the virulence of the $G$. vaginalis strains. Based on the origins of the spacers found in the G. vaginalis CRISPR arrays, we hypothesise that the transfer of genetic material among $G$. vaginalis strains could be regulated by the CRISPR/Cas mechanism. The present study is the first attempt to determine and analyse the CRISPR loci of bacteria isolated from the human vaginal tract.
\end{abstract}

Keywords: Gardnerella vaginalis, Bacterial vaginosis, CRISPR/Cas, Spacer, Repeat, PAM

\section{Background}

Gardnerella vaginalis, a facultatively anaerobic bacterium of the Bifidobacteriaceae family, is strongly associated with bacterial vaginosis (BV): a disease characterised by malodorous vaginal discharge [1-3]. Women with BV are at risk of poor reproductive health outcomes and the acquisition of some sexually transmitted diseases $[2,4]$. BV is defined as a shift in microbial species from hydrogen peroxide producing Lactobacillus to anaerobic bacteria including G. vaginalis, Atopobium vaginae, Prevotella, Peptostreptococcus, and Bacteroides spp. [5,6]. The gold

\footnotetext{
* Correspondence: mildap@ibt.lt

* Correspondence: mildap@ibt.lt
Institute of Biotechnology, Vilnius University, Graiciuno 8, Vilnius LT-02241, Lithuania
}

C B'oWed Central

(c) 2012 Pleckaityte et al.; licensee BioMed Central Ltd. This is an Open Access article distributed under the terms of the Creative Commons Attribution License (http://creativecommons.org/licenses/by/2.0), which permits unrestricted use, distribution, and reproduction in any medium, provided the original work is properly cited. which is used to detr lactobacilli and the bacteria characteristic of BV [7]. The state of asymptomatic BV has also been recognised, although Gram stains revealed a decrease in lactobacilli and an increase in the abundance of anaerobes specific to BV [8]. The same G. vaginalis that is recovered as the prevailing inhabitant of the vaginal tracts of women diagnosed with $\mathrm{BV}$ is also found in BV-negative women, though at much lower numbers $[5,9,10]$. The issue of $G$. vaginalis commensalism is still unclear, as the vaginal bacterial community is dynamic and tends to change during the menstrual cycle to produce transient dominance of G. vaginalis in healthy women $[11,12]$. Using culture- 
independent techniques, it was demonstrated that the vaginal microbiota may differ among human populations: Hispanic and non-Hispanic black women have significantly more anaerobes and fewer lactobacilli than Asian and Caucasian women [12]. Thus, low counts of Lactobacillus do not necessarily indicate the BV state $[6,13]$.

The association of $G$. vaginalis with different clinical phenotypes could be explained by different cytotoxicity of the strains, presumably based on disparities in their gene content. Until recently, surprisingly little has been known about the genetics of G. vaginalis. In 2010, the genomes of several G. vaginalis strains from the vaginas of BV and non-BV patients were sequenced, providing information about the bacterium and enabling comparative genomic analyses [14,15]. Attempts have also been made to expand the knowledge of the genotypic and phenotypic diversity of G. vaginalis strains in terms of virulence factors: particularly vaginolysin, sialidase, and biofilm-forming proteins [16-18]. The development of methods for the genotypic differentiation of $G$. vaginalis revealed that the genomes exhibit great variability. Therefore, some conventional methods, including pulse field gel electrophoresis, restriction fragment length polymorphism, classical ribotyping with Southern blot, and restriction enzyme analysis, are not applicable for typing this species [19-21]. The amplified ribosomal DNA restriction analysis method, while applicable to the genotypic differentiation of G. vaginalis, has been found to not be discriminatory enough for pathogenetic and epidemiological studies of $G$. vaginalis $[17,18]$.

Recent data from G. vaginalis comparative genomic analyses have indicated that the bacterium possesses a small core genome, consisting of 746 genes, that accounts for only $27 \%$ of the pan-genome of the species [22]. The small number of unique genes (21) in the individual strains of G. vaginalis and the genomic plasticity among the strains suggest that horizontal gene transfer (HGT) is active; but there is frequent homologous recombination among G. vaginalis strains, as well as the intake of foreign DNA from other species [15,22].

Clustered Regularly Interspaced Short Palindromic Repeats (CRISPRs) and their associated cas genes constitute a bacterial and archaeal defence mechanism against exogenous nucleic acids [23]. The majority of archaea and approximately half of bacterial genomes contain CRISPR loci [24]. CRISPR loci consist of unique sequences (spacers) that intercalate between short conserved repeat sequences. The spacer sequences often originate from invading viruses and plasmids $[25,26]$. The CRISPR/Cas defence mechanism relies on RNA interference that prevents bacteriophage infection and plasmid conjugation, thus restricting two routes of HGT [27]. Analyses of CRISPR sequences have been used in a variety of applications including strain genotyping and epidemiological study, detection of evolutionary events and bottlenecks, investigation of the history of virus exposure, and host population dynamics, providing insights into the dominant routes of HGT [28-32]. The current study targeted the detection and analysis of CRISPR loci in the genomes of $17 \mathrm{G}$. vaginalis strains isolated from the vaginal tracts of women diagnosed with BV [18], and also in the genomes of $21 \mathrm{G}$. vaginalis strains deposited in the NCBI genome database.

In the current study, we examined the origins of CRISPR spacers representing the immunological memory of G. vaginalis strains, and we hypothesised about the impact of CRISPR/Cas on the emergence of genetic variability of G. vaginalis strains. Also, we demonstrated the restricted distribution of the CRISPR loci among the G. vaginalis strains.

\section{Methods}

\section{G. vaginalis strains}

Seventeen G. vaginalis strains isolated from clinical specimens obtained from the vaginal tracts of women diagnosed with BV were used in this study [18]. The isolates had been previously genotyped/biotyped and characterised with respect to the main known virulence factors, namely vaginolysin and sialidase [18].

Three completely sequenced G. vaginalis genomes (ATCC14019, CP002104.1; 409-05, CP001849.1; and HMP9231, CP002725.1) and 18 G. vaginalis draft genomes were retrieved from the NCBI genome database (http://www.ncbi.nlm.nih.gov/genome/genomes/1967). The accession numbers of the draft genomes are listed in Additional file 1.

\section{CRISPR amplification and sequencing}

Primers for CRISPR amplification were designed by genomic comparison of the CRISPR flanking regions of G. vaginalis strains ATCC 14019, 5-1, AMD, 409-05, $41 \mathrm{~V}, 101$, and 315A. Three different sets of primers; Cas-1-1fw, Cas-3-1fw, CR-1rev, CR-2rev and CR-3rev; were used for the amplification of the CRISPR regions (Additional file 2). PCR was performed in a $50-\mu \mathrm{l}$ reaction mixture containing $0.2 \mu \mathrm{M}$ each primer, $20 \mathrm{ng}$ genomic DNA and 1.5 U Long PCR Enzyme Mix (Thermo Scientific Fermentas, Vilnius, Lithuania). The reaction mixture was subjected to 28 cycles of denaturation at $94^{\circ} \mathrm{C}$ for $30 \mathrm{~s}$, primer annealing at $50^{\circ} \mathrm{C}$ for $40 \mathrm{~s}$, and extension at $72^{\circ} \mathrm{C}$ for $3 \mathrm{~min}$. The final extension step was prolonged to $10 \mathrm{~min}$. PCR products were purified using GeneJET Gel Extraction Kit (Thermo Scientific Fermentas) according to the manufacturer's instructions. The cloned DNA fragments were subjected to sequencing using the $\mathrm{ABI}$ 3130XL genetic analyser. Sequence walking was explored using internal primers constructed within the spacer 
sequences to complete the sequencing of the PCR fragments.

A slightly modified spacer-crawling approach [29] was applied to amplify the CRISPR arrays of strains GV28 and GV33. The primers targeted cas 2 and the repeat sequence within the CRISPR locus. The resulting PCR product represented a ladder consisting of a number of fragments with increasing lengths: each fragment differed by the length of one spacer and one repeat. The mixture of fragments was cloned into the pJET1.2 vector (Thermo Scientific Fermentas); the recombinant plasmids containing the longest DNA inserts were selected and then subjected to sequencing. The next round of amplification used the primer generated from the further spacer sequence and the primers located on the flanking regions downstream of the CRISPR sequence (Additional file 2). The resulting contigs were assembled with a minimum overlapping region of three spacers.

\section{Amplification and sequencing of the cas genes}

The presence of the cas genes was verified by amplification of the regions containing cas5-cas6e-cas1-cas2 ( 3.6 kbp), cas3-cse1 ( 3 kbp), cse2-cas5 ( 2.7 kbp), cas5 ( $0.88 \mathrm{kbp})$ and cse2 ( $\sim 0.6 \mathrm{kbp})$. The primers used in the PCR are provided in Additional file 2. The PCR regimen included 28 cycles of denaturation at $94^{\circ} \mathrm{C}$ for $30 \mathrm{~s}$, primer annealing at $58^{\circ} \mathrm{C}$ for $30 \mathrm{~s}$, and extension at $72^{\circ} \mathrm{C}$ for $1 \mathrm{~min} / \mathrm{kb}$ PCR target. The final extension step was prolonged to $10 \mathrm{~min}$. The cloned DNA fragments containing cas 5 and cas 2 were subjected to sequencing.

\section{CRISPR sequence analysis}

CRISPR information for the three G. vaginalis genomes (ATCC14019, 409-05, and HMP9231) was retrieved from the CRISPR database [24]. CRISPRs Finder [24] was used to detect CRISPR repeat and spacer sequences. The identification of cas genes was also performed using NCBI BLAST (http://blast.ncbi.nlm.nih.gov/Blast.cgi). Each piece of CRISPR and cas information retrieved from the databases was manually proofread. The search for similarities between each spacer and the sequences deposited in GenBank was performed using BLASTn at NCBI, with the search set limited to Bacteria (taxid:2) or Viruses (taxid:10239). All matches with a bit score above 40.0, corresponding to $100 \%$ identity over at least $20 \mathrm{bp}$, were considered legitimate hits. Only the top hit was taken into consideration. Matches to sequences found within G. vaginalis CRISPR loci were discarded. Spacers were compared to one another using the MAFFT program [33]. CRISPR spacers with up to three mismatches that had $100 \%$ overlap between sequences were considered identical. The consensus sequences of the CRISPR repeat and protospacer region alignments were generated by WebLogo [34].

\section{Results}

Architecture of CRISPR/Cas loci in G. vaginalis strains

Two of the three completely sequenced $G$. vaginalis genomes, 12 of the 18 draft genomes in GenBank, and 6 of the $17 \mathrm{G}$. vaginalis clinical isolates contained a cas gene cluster and a CRISPR locus. Sequences consisting of repeats/spacers adjacent to the cas genes were considered CRISPR sequences. The CRISPR/Cas loci in the majority of strains were located between the core gene $c l p C$ and the gene encoding $\mathrm{tRNA}_{\mathrm{Gly}}$ (Figure 1).

The region between the $3^{\prime}$-end of $\operatorname{clp} C$ and the cas genes had ORFs encoding hypothetical proteins and was variable in length $(\sim 5-19 \mathrm{kbp})$, depending on the strain. The region between the 3 -end of the CRISPR array and the gene encoding $\mathrm{tRNA}_{\mathrm{Cys}}$ was not conserved among G. vaginalis strains and varied in length $(0.4-1.8 \mathrm{kbp})$ from strain to strain. The CRISPR/Cas loci of strains 409-05, 00703B, and 00703C2 had different flanking sequences surrounding them. Notably, the region downstream of the CRISPR arrays found in clinical isolates GV21, GV30, GV22, and GV25 corresponded to that found in the genome of the ATCC14019 strain; while the CRISPR flanking sequences on the right, determined in the GV28 and GV33 strains, did not show any similarity to the sequences detected downstream of the G. vaginalis CRISPRs. Due to the variability of the flanking sequences downstream of the CRISPR locus and long CRISPR amplicon, strains GV28 and GV30 contained cas genes but did not produce PCR products. The CRISPR sequences in those two strains were identified using the spacer-crawling approach described in the Methods section. The sequences of the amplified CRISPR regions of six G. vaginalis strains analysed in this study were deposited to GenBank database under the Accession numbers JX215337-JX215342.

The cas loci of $G$. vaginalis consisted of the cas genes cas3-cse1-cse2-cse4-cas5-cas6e-cas1-cas 2 . The detected gene cluster belongs to type I, subtype I-E, known as Ecoli [35]. CRISPR loci were located downstream of cas2 and contained from 1 to 50 spacer sequences. Amplification of the regions containing different cas genes was performed to eliminate false-negative PCRs for CRISPR sequences. PCR products consisting of different sets of cas genes (cas5-cas6e-cas1-cas2, cas3-cse1, cse2-cas5, cas5, and cas 2 ) were obtained from clinical isolates identified as being PCR-positive for CRISPR sequences. The sequences of cas 2 and cas 5 were subjected to sequencing, and their sequences were deposited in GenBank under the Accession numbers JX215343-JX215345.

\section{Characterisation of CRISPR repeat and spacer sequences}

The repeat sequence found in the CRISPR loci of the 20

G. vaginalis strains consisted of 28 bp (Figure 2A), while the spacers in the loci varied in size from 33 to $34 \mathrm{bp}$. 


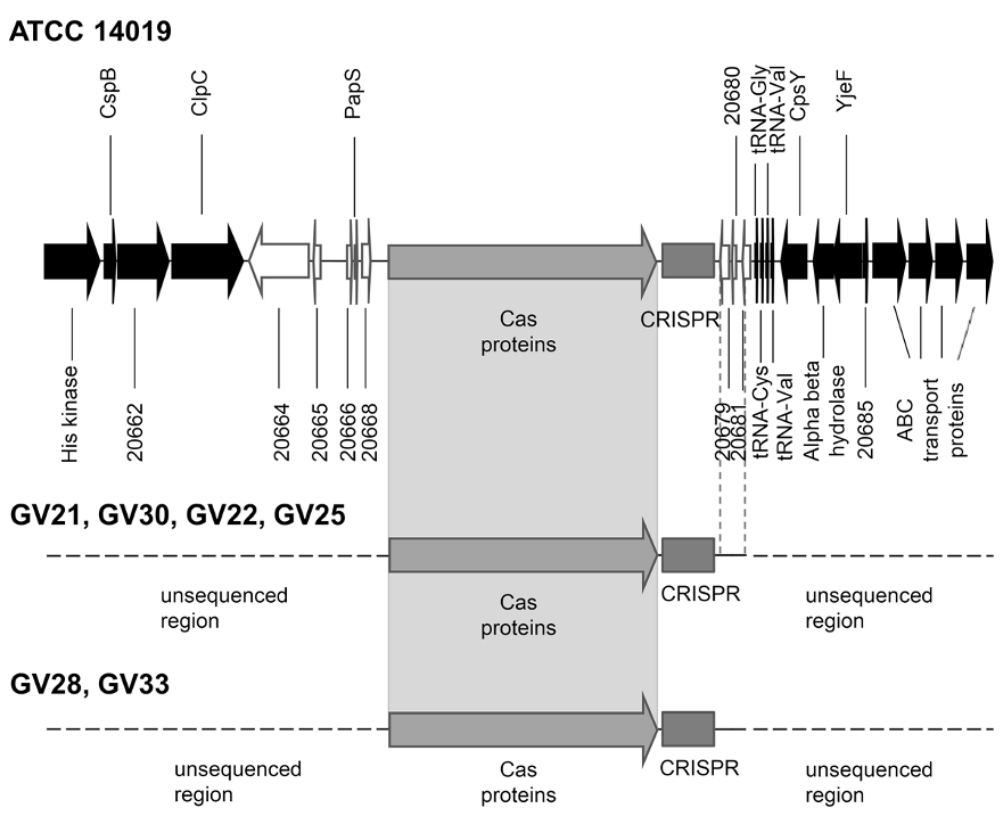

Figure 1 Position of CRISPR/Cas locus on the chromosome of G. vaginalis. The flanking sequence region shared by several strains downstream of the CRISPR array is marked by vertical dashed lines.

The most variable nucleotide positions were found at the proximal ends of the CRISPR repeat (Figure 2A). The repeat sequence of CRISPR was partially palindromic and forms a putative RNA secondary structure with $\Delta \mathrm{G}<-10 \mathrm{kcal} / \mathrm{mol}$ (Figure $2 \mathrm{~B}$ ).

The CRISPR arrays found in the G. vaginalis strains varied in length and spacer content: the longest CRISPR locus contained 40 unique spacers $(40 / 50)$ and was detected in clinical isolate GV25, while only one spacer adjacent to the cas genes was found in strain 1400E. Across six clinical isolates of G. vaginalis, 175 spacers were identified; among them, 129 unique spacers were detected (Figure 3). The fourteen G. vaginalis genomes deposited in GenBank carried 81 unique spacers out of the 110 spacer sequences that were analysed (Figure 3). A total of 285 spacers adjacent to the cas genes were identified among the 20 G. vaginalis strains containing CRISPR/Cas loci (Figure 3).

The trailer-end spacers of the CRISPR loci, i.e. the oldest spacers found farthest from the leader sequences
[37], exhibited several types of conservation: nine strains of G. vaginalis shared one spacer, five strains (among them, the three clinical isolates GV22, GV25, and GV30) shared two spacers, whereas three strains (GV28, 00703B and 00703C2) contained distinct spacer sequence conservation at the trailer -end (Figure 3). All spacer sequences detected within the CRISPR locus of G. vaginalis strain $315 \mathrm{~A}$ had a copy at the trailer-end of clinical isolate GV22 (Figure 3).

\section{Analysis of CRISPR spacer sequences}

All 210 unique spacer sequences were blasted against phage, plasmid, and bacterial sequences. It has been suggested that $100 \%$ identity between spacer and protospacer sequences is required to provide CRISPR-mediated immunity [38]; while the tolerance for mismatches is not yet completely elucidated $[39,40]$. Therefore, a search for protospacers was performed, exploring a less stringent identity criterion by setting a cut-off described in the
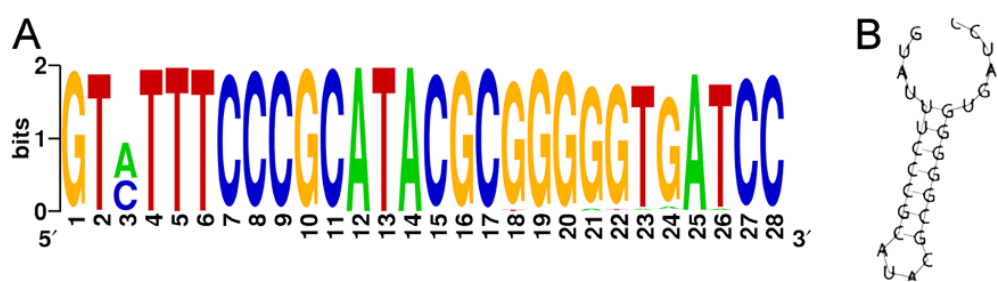

Figure 2 Features of the repeat in the G. vaginalis CRISPR arrays. (A) Sequence logo for all repeats in the CRISPR loci of G. vaginalis. The height of the letters shows the relative frequency of the corresponding nucleotide at that position. (B) Secondary structure of the $G$. vaginalis repeat region predicted using RNAfold [36]. 


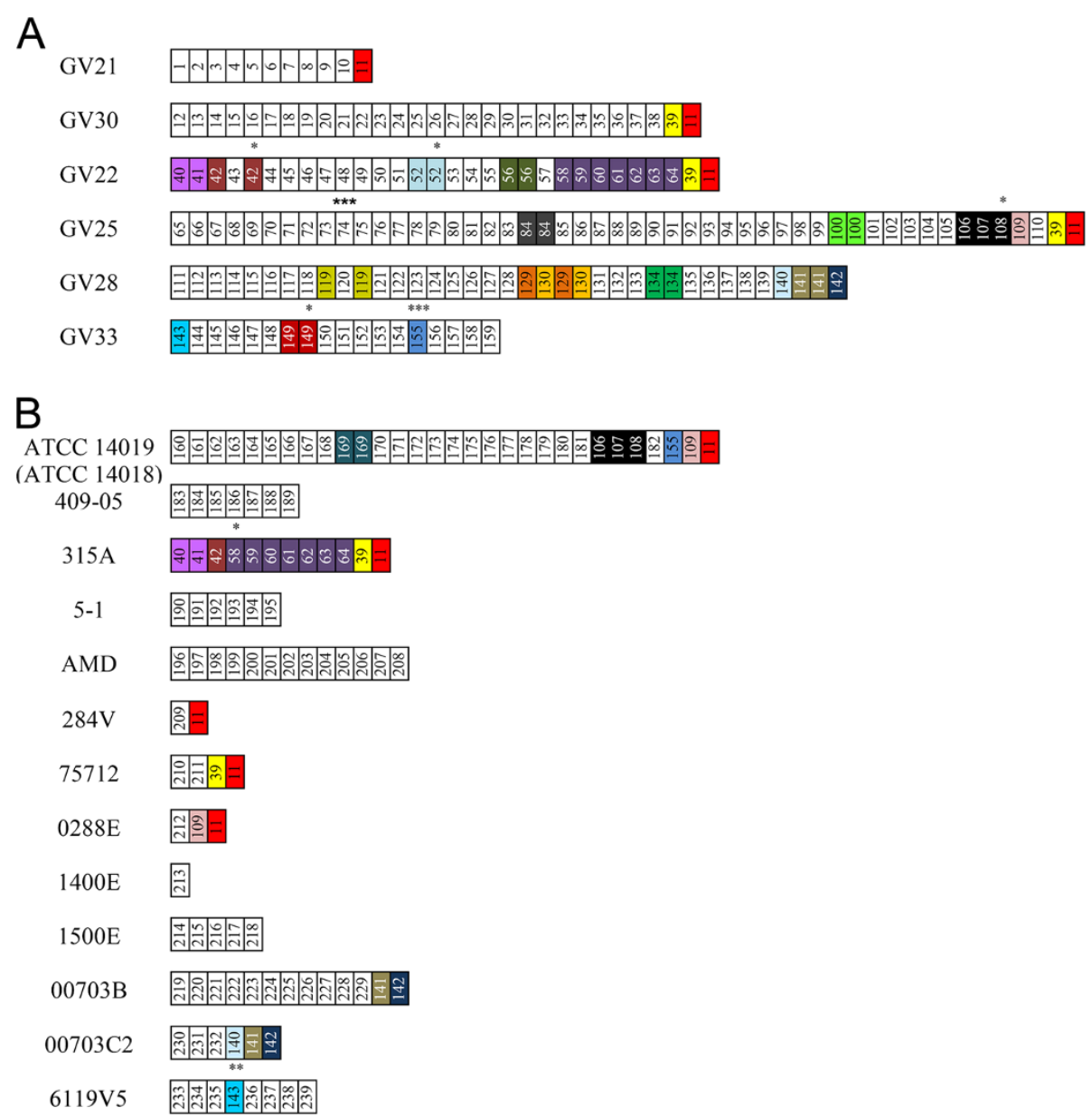

Figure 3 Graphic representation of CRISPR spacers in G. vaginalis clinical isolates (A) and G. vaginalis genomes deposited in GenBank (B). Spacers are represented by boxes; repeats are not included. The leader-end spacers are oriented on the left of each array; the trailer-end spacers are oriented on the right side of each array. Identical spacers are represented by the same number and colour. Unique spacers are whitecoloured. Spacers with mismatches of up to three nucleotides (see Methods) are indicated by dots on the top of the spacer. The number of dots shows the number of mismatched nucleotides.

Methods section. A total of $70.7 \%$ of the spacers had no match to the GenBank database (Figure 4). Overall, among the 70 spacers with matches to the selected cutoff, one sequence showed similarity to a viral sequence, one sequence matched a plasmid sequence, and 68 sequences (97\%) showed similarity to chromosomal sequences (Figure 4; Additional file 3). Among the CRISPR spacers matched to chromosomal sequences of non-G.vaginalis origin, five of 77 spacers were similar to sequences originating from human-associated bacteria including Haemophilus influenza, Weeksella virosa, Campylobacter jejuni, and Bacillus cereus (Additional file 3B). Nearly half of the spacers (32 of 77) were similar to G. vaginalis chromosomal sequences, including 10 spacers that shared 100\% identity (33 of 33 nucleotides; Additional file $3 \mathrm{~A}$ ). All of these spacers, almost uniformly distributed throughout the CRISPR arrays, were unique sequences except for spacer \#106 located at the

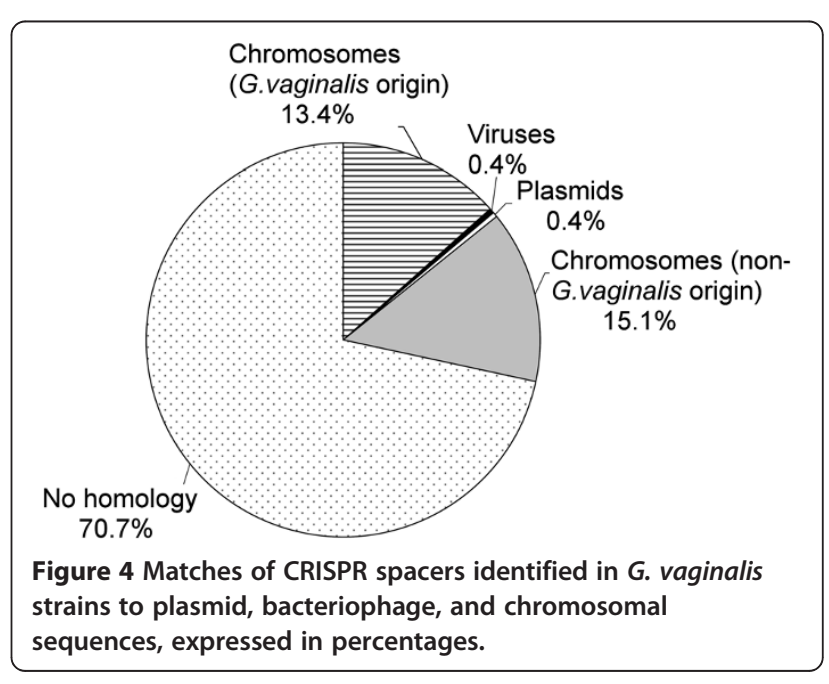


CRISPR trailer-end of strains ATCC14019, ATCC 14018, and GV25.

\section{Spacers matching G. vaginalis chromosomal sequences}

The 28 spacers had significant nucleotide matches to G. vaginalis chromosomal regions ( 85 to $100 \%$ identity), except for three spacers in the CRISPR array of strain $00703 \mathrm{~B}$ and one spacer found in strain GV22 displaying up to $77 \%$ identity (Additional file $3 \mathrm{~A}$ ). Few spacers shared identity with the sequences annotated as having phage origin. Analysis of the G. vaginalis genomes revealed the existence of four to seven phage-associated genes, though most of those were present in one strain and absent in the other strains [15]. We were not able to determine whether the clinical isolates contained the sequences of phage origin targeted by the spacers, because the complete genome sequences are not available yet.

A majority of the spacer hits that mapped to the sequences did not associate with mobile elements (Additional file 3A). The protospacers are localised on both strands of the $G$. vaginalis chromosome, covering coding and non-coding regions. A substantial number of spacers targeting the same region were distributed consecutively in the CRISPR arrays. Nearly $60 \%$ of the CRISPR spacers targeted protospacers located on the chromosome of $G$. vaginalis strain 409-05 (Additional file $3 \mathrm{~A})$. Moreover, different spacers from the CRISPR arrays of different strains targeted the same region of the chromosome. Namely, the spacers in the CRISPR arrays of strains GV22 (one spacer), GV25 (one spacer), GV28 (one spacer), and GV30 (five spacers) clustered in a small $1.1 \mathrm{kbp}$ area and matched the same non-coding region on the chromosome of strain 409-05 (Additional file 3). We did not identify spacers in the CRISPR array of strain 409-05 that shared homology with regions of G. vaginalis chromosomal DNA. Several spacers (\#100 and \#163) originating from different strains targeted the gene encoding $\mathrm{N}$-acetylmuramoyl-L-alanine amidase. None of the CRISPR spacers were found to be self-targeting. We examined the five genomes of $G$. vaginalis available in the NCBI genome database that had spacers targeting coding and non-coding regions on the chromosomes of strains 409-05, 6420B, 315A, 41 V, ATCC14019, and AMD. We did not find a match between the spacers and the endogenous genomic sequences, except for the sequences located in the CRISPR arrays.

We also analysed whether the protospacers located on the G. vaginalis chromosome displayed conserved protospacer adjacent motif (PAM) sequences [41,42]. We aligned the protospacers with the flanking regions comprising 20 bp on both sides. Alignments were performed for ten protospacers sharing $100 \%$ identity with the spacers. The conserved motif of two nucleotides (AA) situated immediately upstream of the target region was detected (Figure 5). The PAM signature AA was confirmed for nine protospacers with up to $10 \%$ mismatches located distant from the $5^{\prime}$ - and $3^{\prime}$-ends of the spacers.

Thus, the motifs adjacent to the protospacers located in the G. vaginalis genomic DNA bear the signatures of PAMs. The orientation of the G. vaginalis PAM is 5'-AA-protospacer-3', which coincides with the orientation of the PAM identified in E. coli as CRISPR/Cas; both bacteria belong to the same type $[41,42]$. Among all of the G. vaginalis CRISPR arrays, the first nucleotide of $97.5 \%$ of the spacers was either $\mathrm{C}$ or T. Only six spacers started with A or G (2.5\%). All of the spacers targeting the protospacers on the $G$. vaginalis chromosome started with $\mathrm{C}$ or $\mathrm{T}(18: 13)$.

\section{Discussion}

The CRISPR locus of the recently discovered CRISPR/ Cas defence system in prokaryotes protects against invading viruses and plasmids and is a map of the "immunological memory" of the microorganism [25,26]. The spacer sequences that are incorporated into the CRISPR loci provide a historical view on the exposure of the bacteria to a variety of foreign genetic elements [23]. A recent report on the ability of CRISPR/Cas to prevent natural transformation in Streptococcus pneumoniae enlarged the role of CRISPR in bacterial nucleic acidbased immunity and the impact that CRISPR has on the emergence of bacterial pathogens [43].

In the current study, we analysed the CRISPR arrays in 17 recently characterised $G$. vaginalis clinical isolates [18] and the genomes of 21 of G. vaginalis strains deposited in the NCBI genome database. We examined the spacer repertoire and evaluated the potential impact of CRISPR/ Cas on gene uptake in G. vaginalis.

We found that six clinical isolates (35\%) and 14 G. vaginalis genomes deposited in the NCBI database (67\%) contained CRISPR/Cas loci. The loci included complete cas genes and repeat sequences interspaced by a variable number of spacers. The repeat sequence in the CRISPR array of $G$. vaginalis was not identical to that found in the E. coli CAS-E subtype [44]. In silico analysis of the Cas proteins revealed highly conserved ( $>97 \%$ identity) sequences among the G. vaginalis strains. The Cas proteins showed the highest similarity (46 to $63 \%$ identity) to the proteins from $A$. vaginae DSM15829 (Ecoli Cas subtype); meanwhile, 9 to $35 \%$ identity was scored to the Cas proteins from E. coli $\mathrm{K} 12$ strain MG1655, which are attributable to the same subtype [35]. The AT-rich leader sequence immediately upstream of the first CRISPR repeat was detected in the genomes of all of the analysed G. vaginalis strains.

Analysis of the spacer repertoire revealed different activities of the CRISPR/Cas loci across different G. vaginalis strains. The CRISPR locus identified in the genome of 


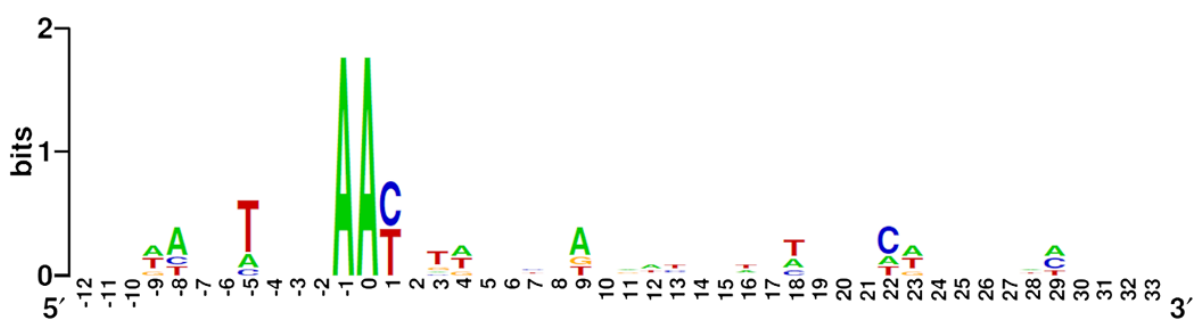

Figure 5 WebLogo for the PAM consensus sequence determination. Ten protospacers identical to spacers were aligned relative to the $5^{\prime}$ end of the protospacer (base 1). Sequences include the protospacer (positive numbers) and 13 nucleotides (negative numbers) upstream of the first base of the protospacer (containing the PAM).

strain GV25 is considered to be the most active, in terms of the degree of spacer polymorphism exhibited by both the total number of unique spacers and the total number of unique spacer arrangements $[38,45]$. In contrast, the spacer content in the CRISPR array of strain 315A could indicate that newly gained CRISPR spacers were deleted and the most ancient spacers were preserved (Figure 3B). We may assume that cas activity in the genome of G. vaginalis strain $315 \mathrm{~A}$ was depleted $[37,45]$.

In the present work, the analysis of CRISPR loci revealed that the majority of CRISPR spacers were similar to chromosomal sequences of both G. vaginalis and non-G.vaginalis origins. Spacer matches to viral and plasmid sequences suggest their putative origin, because there is no evidence of plasmids in the G. vaginalis genomic architecture, and viruses that infect $G$. vaginalis are not yet known $[15,22]$. A substantial portion of the spacers matched G. vaginalis chromosomal sequences. The spacers shared identity with coding and non-coding sequences in the chromosome of G. vaginalis. The spacers were not self-targeting [46], and the protospacers located on the chromosome displayed PAMs. The question of whether $\mathrm{C}$ or $\mathrm{T}$ is the first base of the spacer or the 29th base of the repeat in G. vaginalis CRISPR arrays is still open [46,47]. In our study, all spacers targeting protospacers on the G. vaginalis chromosome started with either $C$ or T. Thus, the spacers correspond to the AAT-PAM or AAC-PAM, assuming that the $\mathrm{C} / \mathrm{T}$ originates from the repeat. Hypotheses about the borders of the CRISPR repeats/spacers need experimental testing; however, the idea of a "duplicon" seems attractive [47].

The analysis of the genomes of G. vaginalis presumed that the chromosomal sequences targeted by spacers did not derive from plasmids or viruses and that the genes in the vicinity of the protospacers (approx. $5 \mathrm{kbp}$ upstream and $5 \mathrm{kbp}$ downstream) do not have viral origin. The gene-coding sequences targeted by the G. vaginalis CRISPR/Cas system were found to not be constituents of mobile-element-associated genes such as restrictionmodification and toxin-antitoxin systems or transposases $[45,48]$. Two spacers from different strains targeted the gene encoding $\mathrm{N}$-acetylmuramoyl-L-alanine amidase: a
CHAP-family domain protein found to have lytic ability [49]. Several strains possess spacers matching the gene encoding the glycoside hydrolase (GH) family 25 protein and the non-coding regions in its close vicinity. The GH 25 family comprises lysozyme able to hydrolyse peptidoglycan and two Abi proteins conferring resistance to a broad range of related bacteriocins $[15,50]$. It has been suggested that these findings are in agreement with the data showing that G. vaginalis strains produce substances antagonistic to bacterial isolates common to the vaginal microbiome [15,51]. A substantial part of the spacers targeted non-coding regions or ORF's encoding hypothetical proteins with undefined functions.

Our data suggest that the CRISPR/Cas system was in touch with $G$. vaginalis DNA that was most probably of chromosomal origin and accessed by the transformation, transduction, or conjugation routes. DNA acquisition and exchange by natural transformation among G. vaginalis strains was detected as a favourable route [22]. Moreover, G. vaginalis strains were found to encode the competence promoting proteins ComEA, ComEC, and CinA [15]; [http://blast.ncbi.nlm.nih.gov/Blast.cgi]. Our data on the origin of the spacers detected in the G. vaginalis CRISPR arrays propose the hypothesis that the transfer of genetic material among $G$. vaginalis strains could be regulated by the CRISPR/Cas mechanism. Circumstances favourable for DNA transfer and CRISPR activity would mean the simultaneous presence of more than one G. vaginalis strain during infection, which is consistent with previous reports $[21,22,52]$. The impact of CRISPR/Cas on the virulence of $G$. vaginalis could involve the spacer targeting the GH family 25 gene that encodes a product promoting competitive exclusion by the 409-05 strain [http://blast. ncbi.nlm.nih.gov/Blast.cgi].

The distribution of CRISPR/Cas loci among pathogenic bacteria that incorporate new genetic material, along with virulence genes, through natural transformation is variable $[27,43]$. The incidence of the CRISPR/Cas system among G. vaginalis strains may be determined by the habitat of the bacteria. The low prevalence of viruses in the human endometrium [53] does not promote the acquisition of CRISPR/Cas by G. vaginalis as an adaptive immunity 
Table 1 G. vaginalis CRISPR spacers and known virulence features

\begin{tabular}{|c|c|c|c|c|c|c|c|c|c|}
\hline \multirow[t]{2}{*}{ Strain } & \multirow[t]{2}{*}{ Reference } & \multirow[t]{2}{*}{ Clinical status } & \multirow[t]{2}{*}{ Biotype } & \multicolumn{2}{|c|}{ Sialidase A } & \multicolumn{2}{|c|}{ Vaginolysin } & \multicolumn{2}{|c|}{ CRISPR } \\
\hline & & & & $\begin{array}{l}\text { Coding } \\
\text { gene }\end{array}$ & Activity & $\begin{array}{l}\text { Coding } \\
\text { gene }\end{array}$ & $\begin{array}{c}\text { Production } \\
\text { level }\end{array}$ & $\begin{array}{c}\text { Number of } \\
\text { spacers }\end{array}$ & $\begin{array}{c}\text { Number of unique } \\
\text { spacers }\end{array}$ \\
\hline ATCC 14019 & {$[15]$} & BV & ND & + & ND & + & $\mathrm{ND}$ & 30 & 24 \\
\hline ATCC 14018 & {$[15]$} & BV & 1 & - & - & + & ND & 30 & 24 \\
\hline 409-05 & {$[15]$} & Asymptomatic BV & ND & - & - & + & ND & 7 & 7 \\
\hline HMP9231 & СР0027525.1 & Not known & ND & + & ND & + & ND & - & - \\
\hline 101 & [14] & BV & ND & + & ND & + & ND & - & - \\
\hline $41 \mathrm{~V}$ & AEJE01000000.1 & Healthy woman & ND & + & ND & + & ND & - & - \\
\hline $315 A$ & AFDI01000000.1 & Not known & ND & + & ND & + & ND & 11 & 0 \\
\hline $5-1$ & [14] & Healthy woman & ND & - & - & + & ND & 6 & 6 \\
\hline AMD & [14] & BV & ND & - & - & + & ND & 13 & 13 \\
\hline $284 \mathrm{~V}$ & [22] & $\begin{array}{l}\text { Abnormal } \\
\text { discharge \& odor }\end{array}$ & 1 & + & ND & + & ND & 2 & 1 \\
\hline 75712 & [22] & BV & 1 & + & ND & + & ND & 3 & 2 \\
\hline 0288E & {$[22]$} & $\begin{array}{l}\text { Abnormal } \\
\text { discharge \& odor }\end{array}$ & 1 & + & ND & + & ND & 3 & 1 \\
\hline 6420LIT & [22] & Healthy woman & 2 & - & - & + & ND & - & - \\
\hline $6420 B$ & [22] & Healthy woman & 2 & - & - & + & ND & - & - \\
\hline 55152 & [22] & Asymptomatic BV & 3 & + & ND & + & ND & - & - \\
\hline $1400 \mathrm{E}$ & [22] & Nugent score 9 & 4 & + & ND & + & ND & 1 & 1 \\
\hline $1500 E$ & [22] & Nugent score 7 & 5 & + & ND & + & ND & 5 & 5 \\
\hline 00703Bmash & [22] & BV & 2 or 5 & + & ND & + & ND & 13 & 11 \\
\hline 00703C2mash & [22] & BV & 2 or 5 & + & ND & + & ND & 6 & 3 \\
\hline 00703Dmash & [22] & BV & 3 or 7 & + & ND & + & ND & - & - \\
\hline $6119 \mathrm{~V} 5$ & [22] & Nugent score 5 & 7 & + & ND & + & ND & 8 & 7 \\
\hline GV15 & [18] & BV & 5 & + & $S$ & + & Low & - & - \\
\hline GV17 & [18] & BV & 5 & + & S & + & Low & - & - \\
\hline GV21 & [18] & BV & 1 & + & W & + & Medium & 11 & 10 \\
\hline GV22 & [18] & BV & 2 & + & - & + & Low & 30 & 13 \\
\hline GV23 & [18] & BV & 1 & + & W & + & High & - & - \\
\hline GV24 & [18] & BV & 1 & + & - & + & Low & - & - \\
\hline GV25 & [18] & BV & 1 & + & W & + & Low & 50 & 40 \\
\hline GV26 & [18] & BV & 1 & + & - & + & Low & - & - \\
\hline GV28 & [18] & BV & 5 & + & $S$ & + & High & 37 & 25 \\
\hline GV29 & [18] & BV & 1 & + & - & + & Low & - & - \\
\hline GV30 & [18] & BV & 1 & + & - & + & Low & 29 & 27 \\
\hline GV31 & [18] & BV & 1 & + & W & + & Medium & - & - \\
\hline GV32 & [18] & BV & 1 & + & - & + & Medium & - & - \\
\hline GV33 & [18] & BV & 5 & + & $S$ & + & Low & 18 & 14 \\
\hline GV34 & [18] & BV & 4 & + & - & + & Low & - & - \\
\hline GV35 & [18] & BV & 5 & + & $S$ & + & Low & - & - \\
\hline GV36 & [18] & BV & 2 & + & S & + & Medium & - & - \\
\hline
\end{tabular}

ND - not done. 
system against foreign DNA. However, the human vagina is a more favourable environment for virus progression, and extravaginal reservoirs have an impact on the distribution of viruses in the vaginal tract [54]. Recent papers have demonstrated that pathogenic bacteria may lose CRISPR/ Cas under certain selective pressure $[55,56]$. The presence of multiple antibiotic resistances is correlated with the loss of CRISPR loci in enterococci [55]. However, we did not find a correlation between the presence of CRISPR/ Cas loci and genes responsible for antibiotic/antimicrobial resistance in $G$. vaginalis strains. In silico analysis of G. vaginalis genomes revealed that strains 14018, 14019, $284 \mathrm{~V}, 315 \mathrm{~A}, 1400 \mathrm{E}, 0288 \mathrm{E}$, and 00703B, all of which possessed CRISPR/Cas, contained genes conferring resistance to bleomycin and methicillin [15]; [http://blast.ncbi.nlm. nih.gov/Blast.cgi]. In addition, G. vaginalis strains 14018 and 14019 contained a gene coding for an aminoglycoside phosphotransferase that increased resistance to aminoglycosides [15]. Selective pressure for virulence other than antibiotic resistance might also have an impact on the presence of CRISPR/Cas loci. In our study, however, the distribution of CRISPR/Cas systems was variable among the $G$. vaginalis strains with elevated virulence potential that were isolated from BV patients (Table 1). Thus, our results did not reveal a potential link between the presence of CRISPR loci and the known virulence features of the strains (Table 1). Overall, our data suggest that CRISPRbased typing does not provide a promising tool for epidemiological discrimination of $G$. vaginalis strains. Moreover, G. vaginalis genomic DNA has exhibited such a great variability [19-22] that the possibility of developing a routine PCR using a set of specific primers for CRISPR loci amplification is doubtful.

The fact that the majority of $G$. vaginalis strains analysed so far were isolated from symptomatic and asymptomatic BV patients, while only few strains were obtained from the vaginas of healthy women, could be an impetus moving forward to elucidate a link between commensal G. vaginalis strains and CRISPR/Cas loci (Table 1). Recent findings on the role of Cas proteins in providing adaptive immunity to bacteria [39,43,57] may motivate experimental testing of hypotheses on how CRISPR/Cas impacts the regulation of the transfer of genetic material among $G$. vaginalis strains.

The present study is the first attempt to determine and analyse CRISPR loci in bacteria isolated from the human vaginal tract. The relationship between prokaryotes and their environment that is recorded in the spacer sequences of CRISPR loci sheds light into the genomic evolution of G. vaginalis.

\section{Conclusions}

The CRISPR/Cas system was detected in the genomes of about one- half of the analysed G. vaginalis strains. The cas genes in the CRISPR/Cas loci of G. vaginalis belong to the Ecoli subtype. A total of 285 spacers adjacent to the cas genes were identified among the $20 \mathrm{G}$. vaginalis strains containing CRISPR/Cas loci. Approximately 20\% of all of the spacers in the CRISPR arrays had matches in the GenBank database. Sequence analysis of the CRISPR arrays revealed that nearly half of the spacers matched G. vaginalis chromosomal sequences. The spacers sharing identity with these chromosomal sequences were determined to not be self-targeting, and presumably were neither a constituent of mobile-element-associated genes nor originated from plasmids/viruses. The spacer hits were mapped to G. vaginalis chromosomal genes, non-coding regions, or ORF's encoding hypothetical proteins with undefined functions. The protospacers located on the $G$. vaginalis chromosome display conserved PAMs. We did not find a link between the presence of CRISPR loci and the known virulence features of G. vaginalis. Based on the origin of the spacers found in the G. vaginalis CRISPR arrays, we hypothesise that the transfer of genetic material among $G$. vaginalis strains could be regulated by the CRISPR/Cas mechanism. Our findings may provide deeper insights into the genetics of G.vaginalis and promote further studies on the role of G. vaginalis in the microbiome of its host.

\section{Additional files}

Additional file 1: Accession numbers of the draft genomes of G.
vaginalis strains used in the study.
Additional file 2: Primers used for CRISPR loci and cas genes
amplification.
Additional file 3: A. Analysis of CRISPR spacers matched to
chromosomal sequences of G. vaginalis origin.
B. Analysis of CRISPR spacers matched to chromosomal sequences of
non-G.vaginalis origin. The table contains information on the spacer
sequences retrieved from the amplified CRISPR loci of G. vaginalis clinical
isolates and from G. vaginalis genomes deposited in GenBank. The
analysis of spacer hits mapped to chromosomal sequences of G. vaginalis
and non-G. vaginalis origin are provided.

\section{Competing interests}

The authors declare no competing interests.

\section{Authors' contributions}

MP carried out primer design and all DNA manipulation procedures, analyzed all results and drafted the manuscript. MZ performed all bioinformatic analysis of CRISPR/Cas system in G. vaginalis genomes. AZ participated in the design of the study and revised the manuscript. All authors read and approved the final manuscript.

\section{Acknowledgements}

We thank Dr. Albertas Timinskas for bioinformatics assistance in the design of primers for CRISPR loci amplification. We are grateful to Prof. Virginijus Siksnys for a critical reading of the manuscript.

Received: 4 October 2012 Accepted: 18 December 2012 Published: 21 December 2012 


\section{References}

1. Catlin BW: Gardnerella vaginalis: characteristics, clinical considerations, and controversies. Clin Microbiol Rev 1992, 5:213-237.

2. Menard JP, Mazouni C, Salem-Cherif I, Fenollar F, Raoult D, Boubli L, Gamerre M, Bretelle F: High vaginal concentrations of Atopobium vaginae and Gardnerella vaginalis in women undergoing preterm labor. Obstet Gynecol 2010, 115:134-140

3. Ferhers K, Twin J, Fowkes FJ, Garland SM, Fehler G, Morton AM, Hocking JS, Tabrizi SN, Bradshaw CS: Bacterial vaginosis (BV) candidate bacteria: associations with $\mathrm{BV}$ and behavioural practices in sexually-experienced and inexperienced women. PLoS One 2012, 7:e30633.

4. Atashili J, Poole C, Ndumbe PM, Adimora AA, Smith JS: Bacterial vaginosis and HIV acquisition: a meta-analysis of published studies. AIDS 2008, 22:1493-1501.

5. Fredricks DN, Fiedler TL, Thomas KK, Oakley BB, Marazzo JM: Targeted PCR of vaginal bacteria associated with bacterial vaginosis. $J$ Clin Microbiol 2007, 45:3270-3276.

6. Turovskiy Y, Sutyak Noll K, Chikindas ML: The aetiology of bacterial vaginosis. J Appl Microbiol 2011, 110:1105-1128.

7. Workowski KA, Berman SM: Centers for disease control and prevention sexually transmitted disease treatment guidelines. Clin Infect Dis 2011, 53:S59-S63.

8. Leitich $\mathrm{H}$, Kiss $\mathrm{H}$ : Asymptomatic bacterial vaginosis and intermediate flora as risk factors for adverse pregnancy outcome. Best Pract Res Clin Obstet Gynaecol 2007, 21:375-390.

9. Kim TK, Thomas SM, Ho M, Sharma S, Reich Cl, Frank JA, Yeater KM, Biggs DR, Nakamura N, Stmpf R, Leigh SR, Tapping Rl, Blanke SR, Slauch JM, Gaskins HR, Weisbaum JS, Olsen GJ, Hoyer LL, Wilson BA: Heterogeneity of vaginal microbial communities within individuals. J Clin Microbiol 2009, 47:1181-1189.

10. Zozaya-Hinchliffe M, Martin DH, Ferris MJ: Quantitative PCR assessments of bacterial species in women with and without bacterial vaginosis. $J$ Clin Microbiol 2010, 48:1812-1819.

11. Srinivasan S, Liu C, Mitchell CM, Fiedler TL, Thomas KK, Agnew KJ, Marazzo $J M$, Fredricks DN: Temporal variability of human vaginal bacteria and relationship with bacterial vaginosis. PLOS One 2010, 5:e10197.

12. Lamont RF, Sobel JD, Akins RA, Hassan SS, Chaiworapsonga T, Kusanovic JP, Romero R: The vaginal microbiome: new information about genital tract flora using molecular based techniques. BJOG 2011, 118:533-549.

13. Forney $L$, Foster $J A$, Ledger $W$ : The vaginal flora of healthy women is not always dominated by Lactobacillus species. J Infect Dis 2006 , 194:1468-1469.

14. Harwich MD Jr, Alves JM, Buck GA, Strauss JF, Patterson JL, Oki AT, Girerd $\mathrm{PH}$, Jefferson KK: Drawing the line between commensal and pathogenic Gardnerella vaginalis through genome analysis and virulence studies. BMC Genomics 2010, 11:375.

15. Yeoman CJ, Yildirim S, Thomas SM, Durkin AS, Torralba M, Sutton G, Buhay CJ, Ding Y, Duhan-Rocha SP, Muzny DM, Qin X, Gibbs RA, Leigh SR, Stumpf $R$, White BA, Highlander SK, Nelson KE, Wilson BA: Comparative genomics of Gardnerella vaginalis strains reveals substantial differences in metabolic and virulence potential. PLoS One 2010, 5:e12411.

16. Patterson JL, Stull-Lane A, Girerd PH, Jefferson KK: Analysis of adherence, biofilm formation and cytotoxicity suggests a greater virulence potential of Gardnerella vaginalis relative to other bacterial vaginosis-associated anaerobes. Microbiology 2010, 156:392-399.

17. Santiago GL, Deschaght $P$, El Aila N, Kiama TN, Verstraelen $H$, Jefferson $K K$, Temmerman M, Vaneechoutte M: Gardnerella vaginalis comprises three genotypes of which two produce sialidase. Am J Obstet Gynecol 2011, 204:450 e1-7.

18. Pleckaityte M, Janulaitiene M, Lasickiene R, Zvirbliene A: Genetic and biochemical diversity of Gardnerella vaginalis strains isolated from women with bacterial vaginosis. FEMS Immunol Med Microbiol 2012, 65:69-77.

19. Wu SR, Hillier SL, Nath K: Genomic DNA fingerprint analysis of biotype 1 Gardnerella vaginalis from patients with and without bacterial vaginosis. J Clin Microbiol 1996, 34:192-195.

20. Ingianni A, Petruzzelli S, Morandotti G, Pompei R: Genotypic differentiation of Gardnerella vaginalis by amplified ribosomal DNA restriction analysis (ARDRA). FEMS Immunol Med Microbiol 1997, 18:61-66.

21. Aroutcheva AA, Simoes JA, Behbakht K, Faro S: Gardnerella vaginalis isolated from patients with bacterial vaginosis and from patients with healthy vaginal ecosystems. Clin Infect Dis 2001, 33:1022-1027.
22. Ahmed A, Earl J, Retchless A, Hillier SL, Rabe LK, Cherpes TL, Powell E, Janto B, Eutsey R, Hiller NL, Boissy R, Dahlgren ME, Hall BG, Costerton JW, Post JC, $\mathrm{Hu}$ FZ, Ehrlich GD: Comparative genomic analyses of seventeen clinical isolates of Gardnerella vaginalis provides evidence of multiple genetically isolated clades consistent with sub-speciation into genovars. J Bacteriol 2012, 194:3922-3937.

23. Horvath $P$, Barrangou R: CRISPR/Cas, the immune system of bacteria and archaea. 2010, 327:167-170.

24. Grissa I, Vergnaud G, Pourcel C: The CRISPRdb database and tools to display CRISPRs and to generate dictionaries of spacers and repeats. BMC Bioinform 2007, 8:172-182.

25. Barrangou R, Fremaux C, Deveau H, Richards M, Boyaval P, Moineau S, Romero DA, Horvath P: CRISPR provides acquired resistance against viruses in prokaryotes. Science 2007, 315:1709-1712.

26. Marraffini LA, Sontheimer EJ: CRISPR interference limits horizontal gene transfer in staphylococci by targeting DNA. Science 2008, 322:1843-1845

27. Marraffini LA: Impact of CRISPR immunity on the emergence of bacterial pathogens. Future Microbiol 2012, 5:693-695.

28. Karginov FV, Hannon GJ: The CRISPR system: small RNA-guided defence in bacteria and archaea. Mol Cell 2010, 37:7-19.

29. Rezzonico F, Smits TH, Duffy B: Diversity, evolution, and functionality of clustered regularly interspaced short palindromic repeat (CRISPR) regions in the fire blight pathogen Erwinia amylovora. Appl Environ Microbiol 2011, 77:3819-3829.

30. Barrangou R, Horvath P: CRISPR: new horizons in phage resistance and strain identification. Annu Rev Food Sci Technol 2012, 3:143-162.

31. Brüggemann $H$, Lomholt HB, Tettelin H, Kilian M: CRISPR/cas loci of type II Propionibacterium acnes confer immunity against acquisition of mobile elements present in type I P. acnes. PLoS One 2012, 7:e34171.

32. Rho M, Wu YW, Tang H, Doak TG, Ye Y: Diverse CRISPR evolving in human microbiomes. PLoS Genet 2012, 8:e1002441.

33. Katoh $\mathrm{K}$, Asimenos $\mathrm{G}$, Toh $\mathrm{H}$ : Multiple alignment of DNA sequences with MAFFT. Methods Mol Biol 2009, 537:39-64

34. Crooks GE, Hon G, Chandonia JM, Brenner SE: WebLogo: a sequence logo generator. Genome Res 2004, 14:1188-1190.

35. Makarova KS, Haft DH, Barrangou R, Brouns SJ, Charpentier E, Horvath $P$, Moineau S, Mojica FJ, Wolf Yl, Yakunin AF, van der Oost J, Koonin EV: Evolution and classification of the CRISPR-Cas systems. Nat Rev Microbiol 2011, 9:467-477.

36. Hofacker I: Vienna RNA secondary structure server. Nucleic Acids Res 2003, 31:3429-3431.

37. Weinberger AD, Sun CL, Pluciński MM, Denef VJ, Thomas BC, Horvath $P$, Barrangou R, Gilmore MS, Getz WM, Banfield JF: Persisting viral sequences shape microbial CRISPR-based immunity. PLOS Comput Biol 2012, 8:e1002475.

38. Horvath $P$, Romero DA, Coûtè-Monvoisin AC, Richards M, Deveau H, Moineau S, Boyaval P, Fremaux C, Barrangou R: Diversity, activity, and evolution of CRISPR loci in Streptococcus thermophilus. J Bacteriol 2008, 190:1401-1412.

39. Sapranauskas R, Gasiunas G, Fremaux C, Barrangou R, Horvath P, Siksnys V: The Streptococcus thermophilus CRISPR/Cas system provides immunity in Escherichia coli. Nucleic Acids Res 2011, 39:9275-9282.

40. Semenova E, Jore MM, Datsenko KA, Semenova A, Westra ER, Wanner B, van der Oost J, Brouns SJ, Severinov K: Interference by clustered regularly interspaced short palindromic repeat (CRISPR) RNA is governed by a seed sequence. Proc Natl Acad Sci USA 2011 108:10098-10103.

41. Mojica FJ, Díez-Villaseñor C, García-Martínez J, Almendros C: Short motif sequences determine the targets of the prokaryotic CRISPR defence system. Microbiology 2009, 155:733-740.

42. Swarts DC, Mosterd C, van Passel MW, Brouns SJ: CRISPR interference directs strand specific acquisition. PLoS One 2012, 7:e35888.

43. Bikard D, Hatoum-Aslan A, Mucida D, Marraffini LA: CRISPR interference can prevent natural transformation and virulence acquisition during in vivo bacterial infection. Cell Host Microbe 2012, 12:177-186.

44. Díez-Villaseñor C, Almendros C, García-Martínez J, Mojica FJ: Diversity of CRISPR loci in Escherichia coli. Microbiology 2010, 156:1351-1361.

45. Touchon M, Rocha EP: The small, slow and specialized CRISPR and anti-CRISPR of Escherichia and Salmonella. PLoS One 2010, 5:e11126.

46. Stern A, Keren L, Wurtzel O, Amitai G, Sorek R: Self-targeting by CRISPR: gene regulation or autoimmunity. Trends Genet 2010, 26:335-340. 
47. Goren MG, Yosef I, Auster O, Qimron U: Experimental definition of a clustered regularly interspaced short palindromic duplicon in Escherichia coli. J Mol Biol 2012, 423:14-16.

48. Brodt A, Lurie-Weinberger MN, Gophna U: CRISPR loci reveal networks of gene exchange in archaea. Biol Direct 2011, 6:65.

49. Bateman A, Rawlings ND: The CHAP domain: a large family of amidases including GSP amidase and peptidoglycan hydrolases. Trends Biochem Sci 2003, 28:234-237.

50. Kjos M, Snipen L, Salehian Z, Nes IF, Diep DB: The Abi proteins and their involvement in bacteriocin self-immunity. J Bacterio/ 2010, 192:2068-2076.

51. Teixeira GS, Soares-Brandão KL, Branco KM, Sampaio JL, Nardi RM, Mendonça M, Almeida RB, Farias LM, Carvalho MA, Nicoli JR: Antagonism and synergism in Gardnerella vaginalis strains isolated from women with bacterial vaginosis. J Med Microbiol 2010, 59:891-897.

52. Piot P, van Dyke E, Peeters M, Hale J, Totten PA, Holmes KK: Biotypes of Gardnerella vaginalis. J Clin Microbiol 1984, 20:667-679.

53. Vestergaard AL, Knudsen UB, Munk T, Rosbach H, Bialasiewicz S, Sloots TP, Martensen PM, Antonsson A: Low prevalence of DNA viruses in the human endometrium and endometriosis. Arch Virol 2010, 155:693-703.

54. Marazzo JM, Fiedler TL, Srinivasan S, Thomas KK, Liu C, Ko D, Xie H, Saracino $M$, Fredricks DN: Extravaginal reservoirs of vaginal bacteria as risk factors for incident bacterial vaginosis. J Infect Dis 2012, 205:1580-1588.

55. Palmer KL, Gilmore MS: Multidrug-resistant enterococci lack CRISPR-cas. mBio 2010, 1:e00227-10.

56. Delaney NF, Balenger S, Bonneaud C, Marx CJ, Hill GE, Ferguson-Noel N, Tsai P, Rodrigo A, Edwards S: Ultrafast evolution and loss of CRISPRs following a host shift in a novel wildlife pathogen, Mycoplasma gallisepticum. PLOS Genet 2012, 8:e1002511.

57. Gasiunas G, Barrangou R, Horvath P, Siksnys V: Cas9-crRNA ribonucleoprotein complex mediates specific DNA cleavage for adaptive immunity in bacteria. Proc Natl Sci USA 2012, 109:E2579-E2586.

doi:10.1186/1471-2180-12-301

Cite this article as: Pleckaityte et al:: Insights into the CRISPR/Cas system of Gardnerella vaginalis. BMC Microbiology 2012 12:301.

\section{Submit your next manuscript to BioMed Central and take full advantage of:}

- Convenient online submission

- Thorough peer review

- No space constraints or color figure charges

- Immediate publication on acceptance

- Inclusion in PubMed, CAS, Scopus and Google Scholar

- Research which is freely available for redistribution

Submit your manuscript at www.biomedcentral.com/submit
() Biomed Central 